Peter Constantin · Komla Domelevo .

Jean-Michel Roquejoffre · Lenya Ryzhik

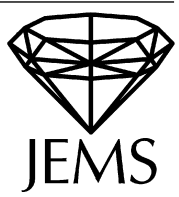

\title{
Existence of pulsating waves in a model of flames in sprays
}

Received June 16, 2005

Abstract. A one-dimensional system describing the propagation of low Mach number flames in sprays is studied. We show that pulsating waves may exist when the droplet distribution in the unburnt region is spatially periodic. The range of possible propagation speeds may be either bounded or unbounded, depending on the threshold temperatures of the burning and vaporization rates.

\section{Introduction and main results}

In view of the breadth of their potential applications and complexity, models for flame propagation in sprays present significant analytical and computational challenges. It is therefore desirable to have a good understanding of the basic phenomena when flame propagation involves both burning of the gas and droplet evaporation that converts them into the flammable gas and provides an additional source of fuel for the flame. We consider a simplified model for the propagation of one-dimensional flames in sprays (see [16] for the background on this model) that involves three unknowns $(T, Y, S)$ :

- $T(t, x)$ is the temperature of the mixture,

- $Y(t, x)$ is the mass fraction of the gaseous reactant,

- $S(t, x)$ represents the surface of the droplets that are, at time $t$, located at $x$. We are thus making the — simplistic but not irrelevant-assumption that, at every point in time and space, the droplets have only one radius. Such a spray is called monodisperse.

The evolution of $T, Y$ and $S$ is described by the following system:

$$
\left\{\begin{array}{l}
T_{t}-T_{x x}=Y f(T), \\
Y_{t}-Y_{x x}=-Y f(T)-\partial_{t}\left(S^{3 / 2}\right), \quad(t, x) \in \mathbb{R}^{2} \\
S_{t}=-\varphi(T) S
\end{array}\right.
$$

P. Constantin: Department of Mathematics, University of Chicago, Chicago, IL 60637, USA; e-mail: const@math.uchicago.edu

K. Domelevo: UMR CNRS 5640 (M.I.P), Université Paul Sabatier, F-31062 Toulouse Cedex, France; e-mail:komla@mip.ups-tlse.fr

J.-M. Roquejoffre: UMR CNRS 5640 (M.I.P), Université Paul Sabatier, F-31062 Toulouse Cedex, France and Institut Universitaire de France; e-mail: roque@mip.ups-tlse.fr

L. Ryzhik: Department of Mathematics, University of Chicago, Chicago, IL 60637, USA;

e-mail: ryzhik@math.uchicago.edu

Mathematics Subject Classification (2000): 35B25 
The first two equations in (1.1) are the usual thermo-diffusive system but for the last term in the equation for $Y$ that represents the gas production due to evaporation of droplets. The last equation describes the decrease in the number of droplets available because of the same process. The reaction rate $f$ and evaporation rate $\varphi$ are smooth, and satisfy the following assumptions:

- $f(0)=\varphi(0)=0$; moreover $f$ and $\varphi$ are nonnegative nondecreasing functions, uniformly bounded from above:

$$
0 \leq \varphi(T), f(T) \leq C \quad \text { for } 0 \leq T<+\infty
$$

- there exist $0 \leq \theta_{v} \leq \theta_{i}<+\infty$ such that $f$ (resp. $\varphi$ ) is positive on $\left(\theta_{i},+\infty\right)$ (resp. $\left.\left(\theta_{v},+\infty\right)\right)$ and zero outside. If $\theta_{v}=0$ we assume $\varphi^{\prime}(0)>0$.

The thresholds $\theta_{v}$ and $\theta_{i}$ are called, respectively, the boiling and ignition temperatures. The ignition temperature can (and sometimes will) be taken equal to 0 .

The system [1.1 has been considered in [7] where travelling fronts have been shown to exist when the density of droplets in the unburnt region is uniform. In reality the droplet distribution is not homogeneous and may be quite complex. As a first step, in this paper we consider a periodic distribution of liquid droplets located "ahead of the front" at $-\infty$ (the convention is that the flame propagates from right to left). This leads to the following boundary conditions as $x \rightarrow-\infty$ :

$$
T(t,-\infty)=0, \quad Y(t,-\infty)=Y_{u}, \quad \lim _{x \rightarrow-\infty}\left(S(t, x)-S_{u}(x)\right)=0,
$$

where

- $Y_{u}$ is a nonnegative quantity, that may-and sometimes will-be 0 ;

- $S_{u}(x)$ is a smooth, positive, 1-periodic function.

The boundary conditions in the burnt region, as $x \rightarrow+\infty$, are as follows. If we askwhich is quite natural-that everything is burnt at $+\infty$, then both $S$ and $Y$ shall be 0 at $+\infty$ :

$$
S(+\infty)=Y(+\infty)=0 .
$$

We will see that the value $T_{b}$ of $T$ as $x \rightarrow+\infty$ will be determined automatically by the values of $Y_{u}$ and the average of $S_{u}(x)$ over the period:

$$
T_{b}=T(+\infty)=Y_{u}+\left\langle S_{u}^{3 / 2}\right\rangle .
$$

Here $\langle g\rangle$ denotes the average of a function $g$ over its period.

As we have mentioned, should the droplet distribution $S_{u}$ at $-\infty$ be constant, the system under study would admit travelling waves [7]. The periodicity of the radius distribution at $-\infty$ leads us to replace this notion by the wider notion of pulsating waves [6, 17], namely solutions of (1.1)-(1.3) that are time-periodic in some galilean reference frame. In more mathematical terms these are solutions of [1.1) of the form $T(t, x)=U(x+c t, x)$ with a function $U$ that is periodic in the second variable. Alternatively, there exists a speed $c>0$ such that

$$
(T, Y, S)(t, x-c t) \text { is } 1 / c \text {-periodic in } t \text {. }
$$

Our first result deals with the case of a positive ignition temperature. 
Theorem 1.1 (Nonzero ignition temperature). Assume $\theta_{i}>0$. There exists a constant $q_{0} \in(0,1)$ such that if

$$
\theta_{v} / \theta_{i} \leq q_{0} \quad \text { and } \quad \max \left(Y_{u}, \min _{x \in[0,1]} S_{u}(x)\right) \geq 1 / q_{0}
$$

then the problem (1.1)-(1.3) has a pulsating wave solution.

The first condition in (1.6) makes sure that the evaporation produces fuel at sufficiently low temperatures on the left. The second assumption means that there is enough fuel, either liquid or gaseous, in the unburnt region. We also show that there exist two constants $c_{0}$ and $c_{1}$ that depend only on the data of the problem so that the pulsating front speed $c$ satisfies

$$
0<\underline{c}_{0} \leq c \leq \bar{c}_{1}<+\infty .
$$

This means that the possible range of speeds is a priori bounded in the ignition case.

The case of a zero ignition temperature is treated separately. Following the terminology of the thermo-diffusive systems we refer to it as the 'KPP case', although the underlying physics, and, potentially, the behaviour of solutions, may vary according to the respective proportion of droplets and gaseous fuel at $-\infty$.

Theorem 1.2. Assume $\theta_{i}=\theta_{v}=0$. There exists $c_{0}>0$ such that the problem (1.1)-1.3) has a pulsating wave solution with the speed $c_{0}$.

It is well known that, in the case of scalar reaction-diffusion equations

$$
u_{t}-u_{x x}=u f(u)
$$

with, for instance, $f>0$ on $[0,1)$ and $f(1)=0$, there exists $c_{0}>0$ such that, for all $c \geq c_{0}$, the above problem has travelling wave solutions that move with the speed $c$ and connect $u=0$ to $u=1$. We would therefore like to see if this is the case here; we have a partial result in this direction.

Theorem 1.3. Assume, for simplicity, that $f(T)=\varphi(T)=T$ for $0 \leq T \leq 1$ and otherwise the aforementioned assumptions are satisfied.

(i) There exists $c_{0}>0$ such that any pulsating wave solution to (1.1)- 1.3 , has a velocity larger than $c_{0}$.

(ii) Assume in addition that $Y_{u}>0$. There exists $c_{1} \geq c_{0}$ such that, for all $c \geq c_{1}$, the problem (1.1-1.3) has a pulsating wave solution that moves with the speed $c$.

We do not know whether $c_{1}=c_{0}$, that is, if the range of speeds is a semi-infinite interval. In particular, we do not know if there exists a pulsating front that moves with the minimal speed. It is also not known whether the velocity spectrum is unbounded when $Y_{u}=0$.

We mention that while existence of pulsating fronts for a single reaction-diffusion equation has been extensively studied [6, 17], the only result for reaction-diffusion systems that we are aware of is that of [15], where small (but nontrivial) perturbations of a planar travelling front have been considered.

The paper is organized as follows. Sections 2 and 3 are devoted to the proof of Theorem 1.1 in Section 2, we perform a priori estimates on potential pulsating wave solutions 
to (1.1)-1.3; in Section 3 we prove the actual existence by a degree argument allowed by the estimates of the preceding section. In Section 4 we prove Theorem 1.2 by approximation by a sequence of problems with ignition temperature. Section 5 is devoted to Theorem 1.3 it is proved via a direct estimate on the velocity, an additional weighted estimate for the temperature and a homotopy argument. Possible extensions are discussed in Section 6 .

\section{The case of nonzero ignition temperature: a priori estimates}

The main difficulty in deriving uniform bounds will be to bound $Y$. Indeed, as opposed to what happens in a purely gaseous flame, there is a positive source term in the equation for $Y$ due to droplet evaporation. This may in turn cause a (mild) unboundedness for the function $Y(t, x)$. We do not see any convincing reason why the Cauchy problem for (1.1) would produce a solution whose $Y$-component would be unbounded, but we are not able to prove it at the moment. It turns out, however, that finding a lower bound for the velocity will help us, because this puts us in the framework of periodic functions with a bounded period $L=1 / c$. This is a strong restriction on the set of global solutions that we have to investigate, and this will help us in finding the upper bounds that will in turn allow us to set up a degree argument.

It also turns out that the bounds that we shall find strongly depend on the value of $Y_{u}$. If $Y_{u}$ is large enough-larger than the ignition temperature $\theta_{i}$-we will be faced with a flame in which the presence of the spray neither helps nor prevents the propagation; the physics of the phenomenon is that of a purely gaseous flame. As opposed to that, when $Y_{u}$ is small or 0 , the propagation cannot take place without the help of the evaporation process; in the limit $Y_{u} \rightarrow 0$ it is really this process that governs the propagation. Thus we are confronted with two very different physics, a situation which is described in detail for travelling waves in [7].

In what follows, we consider a pulsating wave solution $(T, Y, S)$ to $[1.1$, with a speed $c$. System 1.1 is then written in the reference frame of the wave; thus the system under study is now

$$
\left\{\begin{array}{l}
T_{t}-T_{x x}+c T_{x}=Y f(T), \\
Y_{t}-Y_{x x}+c Y_{x}=-Y f(T)-\left(\partial_{t}+c \partial_{x}\right)\left(S^{3 / 2}\right), \quad(t, x) \in \mathbb{R}^{2} . \\
S_{t}+c S_{x}=-\varphi(T) S,
\end{array}\right.
$$

As we have mentioned, the conditions at $+\infty$ for temperature $T$ cannot be chosen arbitrarily. Adding up the equations for $T$ and $Y$, then integrating the whole lot on $(0,1 / c) \times \mathbb{R}$ yields

$$
(T+Y)(+\infty)=Y_{u}+\left\langle S_{u}^{3 / 2}\right\rangle .
$$

This last value will be denoted by $T_{b}\left(Y_{u}, S_{u}\right)$, the burnt gas temperature; these considerations are therefore summed up in the conditions at $\pm \infty$ :

$$
\left\{\begin{array}{l}
T(t,-\infty)=0, \quad T(t,+\infty)=T_{b}\left(Y_{u}, S_{u}\right), \\
Y(t,-\infty)=Y_{u}, \quad Y(t,+\infty)=0, \\
\lim _{x \rightarrow-\infty}\left(S(t, x)-S_{u}(x)\right)=0, \quad S(t,+\infty)=0
\end{array}\right.
$$


and the periodicity condition

$$
(T, Y, S)(t+1 / c, x)=(T, Y, S)(t, x) .
$$

\subsection{A lower bound for the flame speed}

The main result of this section is the following proposition. Hereafter we set

$$
\underline{S}_{u}=\inf _{x \in[0,1]} S_{u}(x) .
$$

Proposition 2.1. If $\max \left(Y_{u}, \underline{S}_{u}\right)$ is large enough, there exists $c_{0}>0$, depending only on the data, such that any solution $(c, T, Y, S)$ of (2.1)-2.3) satisfies $c \geq \underline{c_{0}}$.

Proof. Two cases should be distinguished.

1. The case of large $Y_{u}$. Namely, we assume that

$$
Y_{u}>\theta_{i}
$$

As is classical, introduce the enthalpy function

$$
W(t, x)=T(t, x)+Y(t, x)
$$

we have

$$
W_{t}-W_{x x}+c W_{x}=-\partial_{t}\left(S^{3 / 2}\right) \geq 0, \quad W(t, \pm \infty) \geq Y_{u} .
$$

Letting $t \rightarrow+\infty$ yields $W(t \rightarrow+\infty) \geq Y_{u}$; moreover, this is true for all times as $W$ is periodic in time. Therefore we have

$$
T_{t}-T_{x x}+c T_{x} \geq\left(Y_{u}-T\right) f(T)=: g(T) .
$$

Take any $\delta>0$ such that $Y_{u}-\delta>\left(Y_{u}+\theta_{i}\right) / 2$ and consider the travelling wave $\underline{T}_{\delta}\left(x+c_{\delta} t\right)$ which is a solution of

$$
u_{t}-u_{x x}=g_{\delta}(u), \quad u(t,-\infty)=-\delta, u(t,+\infty)=Y_{u}-\delta .
$$

Here $g_{\delta}(u)=\left(Y_{u}-\delta-u\right) f(u)$ with the convention that $f(u)=0$ for $u<0$. The front speed $c_{\delta}$ is controlled from below by some $\underline{c_{0}}>0$ depending only on $Y_{u}$, the smoothness of $g$ and the size of $\delta$. We claim that $c \geq \bar{c}_{\delta}$; indeed, assume the contrary: this makes $\underline{T}_{\delta}(x)$ a subsolution to $(2.8)$; moreover, due to its limits at $\pm \infty$ we may, up to the correct translation, assume that $\underline{T}_{\delta}(x) \leq T(0, x)$ with a contact point $x_{0}$. The maximum principle implies $T(1 / c, x) \geq \underline{T}_{\delta}(x)$; moreover the periodicity in $t$ implies that $x_{0}$ is still a contact point between $T(1 / c, \cdot)$ and $\underline{T}_{\delta}$. This contradicts the strong maximum principle.

2. The case of large $\underline{S}_{u}$. We give the proof in the case of zero boiling temperature; the case $\theta_{v}>0$ differs only by computational details. As $\phi^{\prime}(0)>0$, there exist $0<\underline{\varphi}<\bar{\varphi}$ such that

$$
\underline{\varphi} T \leq \varphi(T) \leq \bar{\varphi} T \quad \text { on }\left[0, \theta_{i}\right]
$$


By the nonincrease of the lap number for a parabolic equation [1], combined with the fact that $T(t, x)-\theta_{i}$ is a time-global solution for the (appropriately rewritten to subtract $\theta_{i}$ ) first equation of [2.1), there exists a smooth, $c^{-1}$-periodic function $x_{i}(t)$ such that

$$
\forall t \in \mathbb{R}, \quad x<x_{i}(t) \Rightarrow T(t, x)<\theta_{i}, \quad x>x_{i}(t) \Rightarrow T(t, x)>\theta_{i} .
$$

Let $x_{i}^{-}$be the minimum value of $x_{i}$; we may assume it to be 0 . The function $x \mapsto \theta_{i} e^{c x}$ satisfies the equation for $T$ for $x<0$ and is above $T$ for $(t, x) \in \mathbb{R} \times\{0\}$; letting $t \rightarrow+\infty$ implies, after shifting the time origin, $T(t, x) \leq \theta_{i} e^{c x}$ for $(t, x) \in \mathbb{R} \times \mathbb{R}_{-}$. Then we have

$$
S_{t}+c S_{x} \geq-\bar{\varphi} T S \geq-\bar{\varphi} \theta_{i} e^{c x} S, \quad x<0 .
$$

This implies (once again let $t \rightarrow+\infty$ and use the fact that $S$ is $1 / c$-periodic in $t$ )

$$
S(t, x) \geq \underline{S}_{u} \exp \left(-\frac{\bar{\varphi} \theta_{i}}{c^{2}} e^{c x}\right), \quad x<0 .
$$

Our goal is to find a subsolution to the equation for $Y$ in $\mathbb{R} \times \mathbb{R}_{-}$. Note that in this domain $Y$ satisfies

$$
Y_{t}+c Y_{x}-Y_{x x}=\frac{3}{2} \phi(T) S^{3 / 2} \geq \frac{3}{2} \underline{\varphi} T S^{3 / 2} .
$$

To find an explicit subsolution for $Y$ we would be glad to replace $T(t, x)$ by $\theta_{i} e^{c x}$; unfortunately it is an upper bound for $T$, and not a lower bound. To make up for that we first assume $c<1$ (otherwise the proof would end here), then rescale $t$ and $x$ in a parabolic fashion:

$$
\tau=c^{2} t, \quad \xi=c x
$$

Then $T$ satisfies

$$
T_{\tau}-T_{\xi \xi}+T_{\xi}=0 \quad \text { on } \mathbb{R} \times \mathbb{R}_{-} ; \quad T(\tau+c, \xi)=T(\tau, \xi) .
$$

In particular, there exists $\tau_{0} \in[0,1]$ such that $T\left(\tau_{0}+n c, 0\right)=\theta_{i}$ for all $n \in \mathbb{Z}$ : these are the times when the curve $x_{i}(t)$ hits zero. It follows that $T\left(\tau_{0}+n c, \xi\right)>\theta_{i}$ for all $\xi>0$. Without loss of generality we may assume that $\tau_{0}=0$. Moreover, $T$ is a supersolution to the advection equation everywhere. This implies

$$
\forall(t, x) \in[0,1] \times \mathbb{R}_{+}, \quad T(\tau, \xi) \geq \underline{T}(\tau, \xi)
$$

where

$$
\begin{cases}\underline{T}_{\tau}-\underline{T}_{\xi \xi}+\underline{T}_{\xi}=0 & (0<\tau<1, \xi>0) \\ \underline{T}(\tau, 0)=0 & (0<\tau<1) \\ \underline{T}(0, \xi)=\theta_{i} & (\xi>0)\end{cases}
$$

The parabolic Harnack inequality implies the existence of $C>0$ such that

$$
\forall \tau \in[0,1], \quad \underline{T}(\tau, 1) \geq C \theta_{i} .
$$


We may now construct yet another subsolution $\Phi$ for $T$ as

$$
\begin{cases}\Phi_{\tau}-\Phi_{\xi \xi}+\Phi_{\xi}=0 & (0<\tau<1, \xi>0), \\ \Phi(\tau, 1)=C \theta_{i} & (0<\tau<1), \\ \Phi(0, \xi)=\theta_{i} & (0 \leq \xi<1), \\ \Phi(0, \xi)=0 & (\xi<0) .\end{cases}
$$

The maximum principle implies that $\underline{\Phi} \leq T$, hence applying the parabolic Harnack inequality once again we obtain, for a possibly different $C>0$,

$$
\forall \tau \in \mathbb{R}_{+}, \quad T(\tau, 0) \geq C \theta_{i} .
$$

Still working in the $(\tau, \xi)$ variables we end up with (letting $\tau \rightarrow+\infty$ and using the time-periodicity of $T$ )

$$
\forall(\tau, \xi) \in \mathbb{R} \times \mathbb{R}_{-}, \quad T(\tau, \xi) \geq C \theta_{i} e^{\xi} .
$$

Consequently, we obtain, for $(\tau, \xi) \in \mathbb{R} \times \mathbb{R}_{-}$,

$$
Y_{\tau}-Y_{\xi \xi}+Y_{\xi} \geq \frac{C \underline{\varphi} \theta_{i} e^{\xi}}{c^{2}} \underline{S}_{u}^{3 / 2} \exp \left(-\frac{3 \bar{\varphi} \theta_{i}}{2 c^{2}} e^{\xi}\right) .
$$

An eventual subsolution to 2.15 is obtained by setting the time-derivative equal to 0 and solving the differential equation with the zero boundary conditions at $\pm \infty$; recall that the solution of

$$
-u^{\prime \prime}+u^{\prime}=f \quad \text { on } \mathbb{R}_{-}, \quad u(0)=u(-\infty)=0
$$

is

$$
u(\xi)=\int_{\xi}^{0}\left(e^{\xi-\zeta}-e^{\xi}\right) f(\zeta) d \zeta+\left(1-e^{\xi}\right) \int_{-\infty}^{\xi} f(\zeta) d \zeta
$$

Applying formula 2.16 with $f(\xi)=\left(C \bar{\varphi} \theta_{i} e^{\xi} / c^{2}\right) \exp \left(-3 \bar{\varphi} \theta_{i} e^{\xi} / 2 c^{2}\right)$ we obtain

$$
\begin{aligned}
Y(\tau,-1) & \geq C \underline{S}_{u}^{3 / 2} \int_{-\infty}^{-1} \frac{\bar{\varphi} \theta_{i} e^{\zeta}}{c^{2}} \exp \left(-\frac{3 \bar{\varphi} \theta_{i}}{2 c^{2}} e^{\zeta}\right) d \zeta \\
& \geq C \underline{S}_{u}^{3 / 2} \int_{0}^{C / c^{2}} e^{-\eta} d \eta \geq C \underline{S}_{u}^{3 / 2}
\end{aligned}
$$

for a constant $C>0$ under control, as we have assumed that $c<1$.

Come back to the $(t, x)$ variables. As a consequence of 2.17 we have $Y(t,-1 / c) \geq$ $C \underline{S}_{u}^{3 / 2}$, and the function $W(t, x)$ (given by (2.6) ) satisfies

$$
W_{t}-W_{x x}+c W_{x} \geq 0, \quad W(t,+\infty) \geq C\left\langle S_{u}^{3 / 2}\right\rangle, W(t,-1 / c) \geq C \underline{S}_{u}^{3 / 2} .
$$

Therefore, once again, lettting $t \rightarrow+\infty$ and using the periodicity of $W$ in time we conclude that if $\underline{S}_{u}$ is large enough, there is $\delta>0$ such that $W(t, x) \geq \theta_{i}+\delta$ for $x \geq-1 / c$. We conclude as in part 1: let $T^{\delta}$ be a travelling wave solution of

$$
c_{\delta} T_{x}^{\delta}=T_{x x}^{\delta}+\left(\Pi-T^{\delta}\right) f\left(T^{\delta}\right), \quad T^{\delta}(-\infty)=-\delta, T^{\delta}(+\infty)=\Pi,
$$


with $\theta_{i}<\Pi<C \underline{S}_{u}^{3 / 2}$. Then $c_{\delta}$ is bounded below uniformly in $\delta$ for $\delta$ small. Moreover, if $c<c_{\delta}$, then as $\bar{T}_{x}^{\delta}>0$, we have

$$
c T_{x}^{\delta}<T_{x x}^{\delta}+\left(W-T^{\delta}\right) f\left(T^{\delta}\right), \quad x>-1 / c,
$$

and of course,

$$
T_{t}+c T_{x}=T_{x x}+(W-T) f(T) .
$$

Now, shift $T^{\delta}$ to the right so much that $T^{\delta}(x)<T(0, x)$ for all $x$ and start moving it back to the left. There will be $x_{0}$ so that the shifted $T^{\delta}(x)$ and $T(0, x)$ touch for the first time. Then we would still have $T^{\delta} \leq T(0, x)$ everywhere, which would mean that 2.19 ) holds even for $x \leq-1 / c$ simply because $f\left(T^{\delta}\right)=0$ there. Therefore, for this particular shift both $T^{\delta}$ and $T$ touch at exactly one point and $T^{\delta}$ satisfies 2.19 . Then we get a contradiction as in part 1 of this proof.

If $\theta_{v} \neq 0$ we only have to replace $\varphi(T)$ by $\varphi\left(T-\theta_{v}\right)$ for a $\theta_{v}$ slightly above the actual vaporization temperature, and use the same argument.

\subsection{The upper bounds}

This section is devoted to $L^{\infty}$ bounds for all the unknown functions, as well as an upper bound for the front speed. The lower bound for $c$ obtained in the previous section will in particular be of use to us. The result of this section is best stated in terms of the enthalpy function $W$ defined by 2.6 .

Proposition 2.2. Assume that $\theta_{v} \leq q_{0} \theta_{i}$ with a constant $q_{0}<1$. There exists a constant $\bar{W}>0$, depending only on the data, the lower bound $c_{0}$ of Proposition 2.1 and $q_{0}$, such that $\|W\|_{C^{1}\left(\mathbb{R}^{2}\right)} \leq \bar{W}$.

An immediate corollary is

Corollary 2.3. There exists $c_{1}>0$, depending only on the data and $\underline{c_{0}}$, so that $c \leq c_{1}$.

Proof. Assuming Proposition 2.2 to be true, the temperature $T(t, x)$ satisfies

$$
T_{t}-T_{x x}+c T_{x} \leq(\bar{W}-T) f(T), \quad T(t,-\infty)=0, T(t,+\infty) \leq \bar{W} .
$$

Arguing as in Proposition 2.1, part 1, we may prove that, if $\delta>0$ is below $\theta_{i}$ and $\left(c_{1}, \bar{T}(x)\right)$ solves

$$
-\bar{T}^{\prime \prime}+c_{1} \bar{T}^{\prime}=(\bar{W}+\delta-\bar{T}) f(\bar{T}), \quad \bar{T}(t,-\infty)=\delta, T(t,+\infty)=\bar{W}+\delta,
$$

then $c \leq c_{1}$.

Proof of Proposition 2.2. We do not know yet any upper bound for $c$; to make up for that let us come back to the parabolic scaling $\tau=c^{2} t, \xi=c x$. The equation for $W$ is then

$$
W_{\tau}-W_{\xi \xi}+W_{\xi}=\frac{3 \varphi(T) S^{3 / 2}}{2 c^{2}} \text {. }
$$


We wish to find an eventual supersolution for $W$. To do so, let us define $\bar{\xi}$ such that

$$
\begin{aligned}
\forall \tau \in \mathbb{R}, \forall \xi \geq \bar{\xi}, \quad T(\tau, \xi) & \geq\left(T_{b}\left(Y_{u}+\left\langle S_{u}^{3 / 2}\right\rangle\right)+\theta_{i}\right) / 2 \\
W(\tau, \xi) & \leq 2 T_{b}\left(Y_{u}, S_{u}\right)
\end{aligned}
$$

If $x_{i}(t)$ is defined by 2.10$)$, let $\xi_{i}(\tau)$ be its counterpart in the $(\tau, \xi)$ system. Without loss of generality we may assume that the function $\xi_{i}$ takes its minimum at $\xi=0$. Two cases are to be discussed.

The region $\xi \leq 0$. We simply have $T(\tau, \xi) \leq \theta_{i} e^{\xi}$, hence

$$
\varphi(T) S^{3 / 2} \leq C\|S\|_{\infty}^{3 / 2} \theta_{i} e^{\xi}
$$

The region $\xi \geq 0$. Let us find an upper bound for $S^{3 / 2} \varphi(T)$. We have (see the proof of Proposition 2.1) a constant $q_{1} \in(0,1]$ such that

$$
\forall \tau \in \mathbb{R}, \forall \xi>0, \quad T(\tau, \xi) \geq q_{1} \theta_{i}
$$

We take $q_{0}<q_{1}$. Then there is $\bar{\varphi}>0$ such that

$$
\forall \tau \in \mathbb{R}, \forall \xi>0, \quad \varphi(T(\tau, \xi)) \geq \bar{\varphi}
$$

An eventual supersolution for $S$ in $\{\xi \geq 0\}$ is

$$
\bar{S}(\tau, \xi)=\|S\|_{\infty} e^{-\bar{\varphi} \xi / c^{2}}
$$

This will bound $S(\tau, \xi)$ for $\xi \geq 0$. Gathering 2.23 and 2.24 we realize that there is $C>0$ depending only on the data such that

$$
\forall \tau>0, \quad \frac{1}{c^{2}}\left\|S^{3 / 2}(\tau) \varphi(T(\tau))\right\|_{L_{\xi}^{1}(\mathbb{R})} \leq C
$$

An eventual supersolution for $W$ is the function $\bar{W}(\xi)$ which satisfies, on $\{\xi \leq \bar{\xi}\}$,

$$
-\bar{W}^{\prime \prime}+\bar{W}^{\prime}=\frac{C}{c^{2}}\|S\|_{\infty}^{3 / 2} \theta_{i} e^{\xi} \mathbf{1}_{\mathbb{R}_{-}}+\frac{\|\varphi\|_{\infty}\|S\|_{\infty}^{3 / 2} e^{-\bar{\varphi} \xi / c^{2}}}{c^{2}}
$$

with $\bar{W}(-\infty)=Y_{u}$ and $\bar{W}(\bar{\xi})=2 T_{b}\left(Y_{u}, S_{u}\right)$. A simple ODE integration shows that $\bar{W}(\xi)$ is bounded independently of $c$. The $C^{1}$ bounds follow from parabolic regularity. 


\subsection{Uniform exponential decay}

Assume that the solution $(c, T, Y, S)$ of 2.1]-2.3 additionally satisfies the normalization condition

$$
T(0,0)=\theta_{i} .
$$

The main result of this subsection is the following.

Proposition 2.4. There exist $\rho_{0}>0$ and $C>0$, depending only on the data, such that, for all $t \in \mathbb{R}$,

$$
\begin{array}{ll}
\forall x \in \mathbb{R}_{-}, & \left|\left(T(t, x), Y(t, x)-Y_{u}, S(t, x)-S_{u}(x-c t)\right)\right| \leq C e^{\rho_{0} x}, \\
\forall x \in \mathbb{R}_{+}, & \left|\left(T(t, x)-Y_{u}-\left\langle S_{u}^{3 / 2}\right\rangle, Y(t, x), S(t, x)\right)\right| \leq C e^{-\rho_{0} x} .
\end{array}
$$

By parabolic regularity, it is sufficient to prove the following

Lemma 2.5. There exist $\rho_{0}>0$ and $C>0$, depending only on the data, such that, for all $t \in \mathbb{R}$,

$$
\begin{aligned}
& \left\|e^{-\rho_{0} x}\left(T(t, \cdot), Y(t, \cdot)-Y_{u}, S(t, \cdot)-S_{u}(\cdot-c t)\right)\right\|_{L^{2}\left(\mathbb{R}_{-}\right)} \leq C, \\
& \left\|e^{\rho_{0} x}\left(T(t, \cdot)-Y_{u}-\left\langle S_{u}^{3 / 2}\right\rangle, Y(t, \cdot), S(t, \cdot)\right)\right\|_{L^{2}\left(\mathbb{R}_{+}\right)} \leq C .
\end{aligned}
$$

An important intermediate step is

Lemma 2.6. Let $x_{i}(t)$ be the function defined by 2.10]. There is $\bar{x}_{0}>0$ such that $\left|x_{i}(t)\right| \leq \bar{x}_{0}$ for all $t \in \mathbb{R}$.

Proof of Lemma 2.6 Let $-x_{0} \leq 0$ be the minimal value of $x_{i}$; the proof of Proposition 2.1 (part 2) yields the existence of $C>0$ such that $T\left(t,-x_{0}\right) \geq C \theta_{i}$. Also, remember the existence (as in the proof of Proposition 2.1p of $\underline{W}>\theta_{i}$, only depending on the data, such that $W(t, x) \geq \underline{W}$. Consequently, we have

$$
T_{t}-T_{x x}+c T_{x} \geq(\underline{W}-T) f(T), \quad T(t,+\infty) \geq \underline{W} .
$$

For any $W>\theta_{i}$, let $c(W)$ be the unique speed of the travelling wave connecting 0 to $W$ by the equation

$$
-u^{\prime \prime}+c u^{\prime}=(W-u) f(u) .
$$

Two cases are to be investigated.

- If $c \leq c(\underline{W})$, then there exists (see [4]) a unique solution $\underline{T}(x)$ to

$$
\begin{aligned}
& -\underline{T}^{\prime \prime}+c \underline{T}^{\prime}=(\underline{W}-\underline{T}) f(\underline{T}) \quad\left(x>x_{0}\right), \\
& \underline{T}\left(x_{0}\right)=C \theta_{i}, \quad \underline{T}(+\infty)=\underline{W},
\end{aligned}
$$

which is an eventual subsolution to 2.29]. Moreover, because of the boundedness of $c$ from above, there is an absolute constant $\rho_{0}>0$ such that

$$
\forall x>x_{0}, \quad|\underline{T}(x)-\underline{W}| \leq C e^{-\rho_{0}\left(x-x_{0}\right)} .
$$


- If $c \geq c(\underline{W})$, then (see Aronson-Weinberger [2], [3]) we have

$$
T(t, 0) \geq \underline{W} .
$$

Then $Y$ decays, in both cases, exponentially to 0 as $x \rightarrow+\infty$, at a uniform rate. This implies an inequality for $T$ of the form (2.32).

All this is enough to prove the lemma: the minimal value $-x_{0}$ cannot be too negative since $T(0,0) \geq \theta_{i}$, and the maximal value of $x_{i}(t)$ is bounded directly by $(2.32)$.

Proof of Lemma 2.5. Once $x_{0}$ is known to be bounded, the first part of (2.29) - the bounds on the left-is easy: indeed, we have $0 \leq T(t, x) \leq \theta_{i} e^{c\left(x-x_{0}\right)}$; this is enough due to the uniform boundedness of $c$ from below. Then, using the boundedness of $S$ by $\left\|S_{u}\right\|_{\infty}$ we have the existence of a constant $C>0$ such that

$$
\left|Y_{t}-Y_{x x}+c Y_{x}\right| \leq C e^{c\left(x-x_{0}\right)} ;
$$

an eventual, exponentially decreasing supersolution is easily found and left to the reader. The equation for $S$ is treated in the same fashion.

Consider any $\delta>0$. From Lemma 2.6, there is $x_{1}>0$, uniformly bounded, such that $T \geq \theta_{i}+\delta$ as soon as $x \geq x_{1}$. This forces an exponential decay for $S$ due to the boundedness of $c$ from below; this in turn forces an exponential decay for $Y$ from the maximum principle. It remains to prove the $L^{2}$ bound for $W$; to do so we argue as follows. First, for convenience, rescale the time: $\tau=c t$; the new function $W$ is hence 1-periodic in $t$. Decompose $W(\tau, x)$ in a Fourier series:

$$
W(\tau, x)=\sum_{n \in \mathbb{Z}} w_{n}(x) e^{2 i \pi n \tau} .
$$

The equation for $w_{0}(x)$ is

$$
-w_{0}^{\prime \prime}+c w_{0}^{\prime}=\frac{3}{2} \int_{0}^{1} \varphi(T) S(\tau, x)^{3 / 2} d \tau,
$$

which implies

$$
w_{0}(x)=T_{b}\left(Y_{u}, S_{u}\right)-\frac{3}{2} \int_{x}^{+\infty} e^{c(x-y)} \int_{-\infty}^{y} \int_{0}^{1} \varphi(T) S(\tau, z)^{3 / 2} d \tau d z d y .
$$

The limits for $w_{0}$ are $Y_{u}$ and $T_{b}\left(Y_{u}, S_{u}\right)$, because $3 \varphi(T) S(\tau, z)^{3 / 2} / 2$ is exactly equal to $-\left(\partial_{t}+\partial_{x}\right) S^{3 / 2}$; the desired $L^{2}$ bound for $w_{0}$ is obtained, at the possible expense of decreasing $\rho_{0}$ a bit, by recalling the exponential decay of $S$ at $+\infty$ and the fact that $\varphi(T)$ vanishes for large negative $x$.

For $n \neq 0$ the equation for $w_{n}$ is

$$
\begin{aligned}
-w_{n}^{\prime \prime}+c w_{n}^{\prime}+2 i \pi c n w_{n} & =-\left(2 i \pi c n+c \partial_{x}\right) \int_{0}^{1} e^{-2 i \pi n \tau} S(\tau, x)^{3 / 2} d \tau \quad(x \in \mathbb{R}), \\
w_{n}( \pm \infty) & =0 .
\end{aligned}
$$


This equation has two characteristic roots $r^{ \pm}$, whose real part is above (resp. below) $C(1+\sqrt{n})($ resp. $-C(1+\sqrt{n}))$ where $C$ is a constant under uniform control. This implies, by an elementary computation, the existence of a small, uniform $\rho>0$ such that

$$
\left\|e^{\rho_{0}|x|} w_{n}\right\|_{L^{2}(\mathbb{R})} \leq C / n .
$$

This in turn implies an $L^{2}\left([0,1], L^{2}(\mathbb{R})\right)$ bound for $e^{\rho_{0} x} W(t, x)$.

\section{Nonzero ignition temperature: construction of the wave}

The uniform $L^{2}$ bounds for $W$ and $T$ will allow us to perform directly a topological degree argument for the system (2.1)-2.3) on the whole real line, without the approximation step on a finite interval taken in [5]. The system will first be reduced to a fixed point problem; then we shall introduce a homotopy bringing it to the problem of finding a travelling wave for the 1D thermo-diffusive scalar equation-for which everything is known.

\subsection{Strategy}

To explain how we wish to proceed, let us start by recalling some basic facts. Assume that we are given a Banach space $X$ and a sectorial operator $A$ such that $\left\|e^{-A}\right\|<1$. For $\alpha \in(0,1)$, consider a function $f(t) \in C^{\alpha}(\mathbb{R}, X)$ which is 1-periodic. The Cauchy problem for

$$
\dot{u}+A u=f(t)
$$

is well-posed, in the sense that, for every initial datum $u_{0} \in X$, it admits a unique strong solution $u(t)$ such that $u(0)=u_{0}$. We are interested in finding some 1-periodic solutions for 3.1); to do so it is sufficient to look for the initial datum; it is uniquely given by

$$
u_{0}=\left(I-e^{-A}\right)^{-1} \int_{0}^{1} e^{-(1-s) A} f(s) d s,
$$

and the (unique) 1-periodic solution of 3.1 is given by

$$
u(t)=e^{-t A}\left(I-e^{-A}\right)^{-1} \int_{0}^{1} e^{-(1-s) A} f(s) d s+\int_{0}^{t} e^{-(t-s) A} f(s) d s .
$$

Let us denote by $\mathcal{F} f$ the right side of 3.2. If the right side of 3.1) is replaced by a nonlinear function $f(t, u)$ which is, say, Hölder in its first variable and Lipschitz in its second variable and which is moreover 1-periodic in time, the problem of finding periodic solutions to

$$
\dot{u}+A u=f(t, u)
$$

reduces to

$$
u=\mathcal{F} f(\cdot, u) .
$$

It is this very simple fact that we wish to use in order to reduce 2.1)-(2.3) to a fixed point problem, the major point that we will have to care about being that $u \mapsto \mathcal{F} f(\cdot, u)$ should be compact if we wish to have a chance to apply a degree argument. 


\subsection{Fixed point setting}

Let us try to apply the above strategy, namely to define a subspace $X$ of $C_{\mathrm{per}}^{\alpha, \alpha / 2}\left(\mathbb{R}^{2}\right) \times$ $C_{\text {per }}^{\alpha, \alpha / 2}\left(\mathbb{R}^{2}\right) \times C_{\text {per }}\left(\mathbb{R}^{2}\right) \times \mathbb{R}$ and a compact nonlinear mapping $\mathcal{F}$ of $X$ such that 2.1]-2.3 reduces to finding a fixed point of $\mathcal{F}$. Here the subscript per means that we are dealing with functions of the variables $(t, x)$ that are 1-periodic in $t$.

In what follows, we take the boiling and ignition temperatures $\theta_{i}$ and $\theta_{v}$ to be positive, in agreement with the assumption $\theta_{i}>0$ of Theorem 1.1. The other data are also assumed to be in agreement with the assumptions of this theorem. The limit $\theta_{v} \rightarrow 0$ will be considered at the end of this section.

The first step is to renormalize the time so that the period in 2.3 becomes 1 . We take $Y, W, S, c$ as our principal unknowns instead of $T, Y, S, c$; the reason for this choice will become clear as the discussion goes on. The set of equations that have to be satisfied then becomes

$$
\left\{\begin{array}{l}
c Y_{t}-Y_{x x}+c Y_{x}=-Y f(W-Y)-c\left(\partial_{t}+\partial_{x}\right)\left(S^{3 / 2}\right), \\
c W_{t}-W_{x x}+c W_{x}=-c\left(\partial_{t}+\partial_{x}\right)\left(S^{3 / 2}\right), \\
S_{t}+S_{x}=-\frac{1}{c} \varphi(W-Y) S
\end{array} \quad(t, x) \in \mathbb{R}^{2},\right.
$$

together with the conditions at $\pm \infty$,

$$
\left\{\begin{array}{l}
Y(t,-\infty)=Y_{u}, \quad Y(t,+\infty)=0 \\
W(t,-\infty)=Y_{u}, \quad W(t,+\infty)=T_{b}\left(Y_{u}, S_{u}\right) \\
\lim _{x \rightarrow-\infty}\left(S(t, x)-S_{u}(x)\right)=0, \quad S(t,+\infty)=0
\end{array}\right.
$$

the periodicity condition

$$
(Y, W, S)(t+1, x)=(Y, W, S)(t, x)
$$

and the normalization condition

$$
(W-Y)(0,0)=\theta_{i} .
$$

Let us then define the space $X_{r}$ as

$$
X_{r}=\left\{u \in C_{\mathrm{per}}^{\alpha, \alpha / 2}: e^{r|x|} u \in C_{\mathrm{per}}^{\alpha, \alpha / 2}\right\} .
$$

We first choose, once and for all, $\alpha \in(0,1)$ which will measure the Hölder character of $Y$ and $W$. Next, we recall the real number $\rho_{0}$ defined in Lemma 2.5 , the lower bound $\underline{c}_{0}$ for the velocity, its upper bound $\bar{c}_{0}$, and we fix $r>0$ such that

$$
r<\frac{1}{5} \min \left(\rho_{0}, \underline{c_{0}}, \frac{-\bar{c}_{0}+\sqrt{\bar{c}_{0}^{2}+4 f\left(T_{b}\left(Y_{u}, S_{u}\right)\right)}}{2}\right) .
$$


In fact, the function $S$ will not be a principal unknown: we will compute it directly from $W$ and $Y$. Assume therefore that $Y$ and $W$ are known; then the equation for $S$ in (3.4) has a unique 1-periodic solution in $t$ that is given by

$$
S(t, x)=S_{u}(x-t) \exp \left(-\frac{1}{c} \int_{-\infty}^{0} \varphi_{4}(t+s, x+s) d s\right)
$$

where we have set $\varphi_{4}(s, y)=\varphi(W-Y)(s, y)$. We denote the right side of 3.10 by $\mathcal{F}_{4}(c, Y, W)(t, x)$.

Let $\gamma(x)$ be a smooth nonnegative function that is equal to 0 on $(-\infty,-1]$ and to 1 on $\mathbb{R}_{+}$. In order to obtain unknowns that decay exponentially at $\pm \infty$, let $u_{0}(x)$ and $w_{0}(x)$ be defined as

$$
u_{0}(x)=Y_{u}(1-\gamma(x)), \quad w_{0}(x)=Y_{u}+\left\langle S_{u}^{3 / 2}\right\rangle \gamma(x)
$$

Then look for $Y$ and $W$ in the form

$$
Y=u_{0}+u, \quad W=w_{0}+w .
$$

Examination of 3.10 and of the definition of $\rho_{0}$ in Lemma 2.5 yields the following estimate for $\mathcal{F}_{4}$ :

Lemma 3.1. Consider two functions $Y$ and $W$ of the form (3.12) with $u, w$ in a bounded subset of $X_{r}$, and $c \in\left[\underline{c_{0}} / 2,2 \bar{c}_{0}\right]$. Then $\mathcal{F}_{4}(c, Y, W)(t, x)$ has the form

$$
\mathcal{F}_{4}(c, Y, W)(t, x)=S_{u}(x-t)(1-\gamma(x))+\tilde{\mathcal{F}}_{4}(c, Y, W)(t, x)
$$

with $\tilde{\mathcal{F}}_{4}(c, Y, W) \in X_{r}$; moreover there is a constant $C(c, Y, W)>0$ such that

$$
\left|\tilde{\mathcal{F}}_{4}(c, Y, W)(t, x)\right| \leq C e^{-\rho_{0}|x|} .
$$

Next we turn to the equation for $Y$. The first equation in 3.4 is rewritten as (for short we redefine $T$ as $W-Y$, and we keep the notations $W$ and $Y$ when we do not want to underline a specific decay property)

$$
\begin{aligned}
c u_{t}-u_{x x}+c u_{x}+ & \gamma(x) f\left(T_{b}\left(Y_{u}, S_{u}\right)\right) u \\
= & -\left(f(T)-\gamma(x) f\left(T_{b}\left(Y_{u}, S_{u}\right)\right) Y-\gamma(x) f\left(T_{b}\left(Y_{u}, S_{u}\right)\right) u_{0}\right. \\
& -c\left(\partial_{t}+\partial_{x}\right) \mathcal{F}_{4}(c, Y, W)^{3 / 2}+u_{0}^{\prime \prime}-c u_{0}^{\prime} .
\end{aligned}
$$

Let $A_{c}$ denote the differential operator

$$
A_{c}=-c^{-1} \frac{d^{2}}{d x^{2}}+\frac{d}{d x}+c^{-1} \gamma(x) f\left(T_{b}\left(Y_{u}, S_{u}\right)\right) .
$$

If $U C(\mathbb{R})$ is the space of all bounded, continuous functions of $\mathbb{R}$, we define, for all $\rho>0$,

$$
Y_{\rho}=\left\{u \in U C(\mathbb{R}): e^{-\rho|x|} u \in U C(\mathbb{R})\right\} .
$$

We extract from (3.9) and [9. Chap. 5] the following 
Lemma 3.2. There is $\delta_{0}>0$, depending on $\underline{c}_{0}$ and $\rho_{0}$, such that, for every $c \in\left[\underline{c_{0}} / 2,2 \bar{c}_{0}\right]$, and for all $\delta \in\left[0, \delta_{0}\right]$, the operator $A_{c}$ is sectorial in $Y_{r+\delta_{0}}$; its spectrum is moreover in the right complex half-plane, bounded away from the imaginary axis. This statement is uniform with respect to $\delta \in\left[0, \delta_{0}\right]$.

The proof is standard and omitted. Thus $e^{-A_{c}}$ has norm $<1$ in $X_{r+\delta}$ for every $\delta \in\left[0, \delta_{0}\right]$; hence we may define $\mathcal{F}_{2}(c, u, w)$ as follows:

- the underlying space is $Y_{r}$;

- the quantity $\mathcal{F}_{2}(c, u, w)$ is defined by (3.2), with $f$ being the RHS of (3.13).

Rephrasing Lemma 2.5, we have

Lemma 3.3. For the quantity $\delta_{0}$ of Lemma 3.2 the mapping $(c, u, w) \mapsto \mathcal{F}_{2}(c, u, w)$ is $C^{1}$ and compact from $\left[\underline{c_{0}} / 2,2 \bar{c}_{0}\right] \times X_{r} \times X_{r}$ into $X_{r+\delta_{0}}$.

This implies the following

Proposition 3.4. The mapping $\mathcal{F}_{2}$ is $C^{1}$ and compact from $\left[\underline{c_{0}} / 2,2 \bar{c}_{0}\right] \times X_{r} \times X_{r}$ into $X_{r}$.

Proof. Straightforward, but lengthy_-by Lemma 3.3 and parabolic regularity. We omit it.

We would now like to do the same operation for the $W$-equation, but we do not have here a term that ensures some coercivity at $+\infty$. However, if we set, for all $\rho>0$,

$$
\tilde{Y}_{\rho}=\left\{u \in U C(\mathbb{R}): e^{-\rho x} u \in U C(\mathbb{R})\right\}
$$

then, for all $c \in\left[\underline{c_{0}} / 2,2 \bar{c}_{0}\right]$, the operator

$$
B_{c}=-c^{-1} \frac{d^{2}}{d x^{2}}+\frac{d}{d x}
$$

satisfies $\left\|e^{-B_{c}}\right\|_{\mathcal{L}\left(\tilde{Y}_{r}\right)}<1$, uniformly in $c$. The verification is even simpler than for Lemma 3.2. and is therefore omitted. Consequently, a mapping can be constructed as before: first, the equation for $w$ is

$$
c w_{t}-w_{x x}+c w_{x}=-c\left(\partial_{t}+\partial_{x}\right)\left(\mathcal{F}_{4}(c, Y, W)\right)^{3 / 2}-w_{0}^{\prime \prime}+c w_{0}^{\prime} .
$$

Then, by writing formula (3.2) with $f$ as the RHS of 3.14), and $A=B_{c}$, we obtain a mapping that we call $\mathcal{F}_{3}(c, Y, W)$. Now, Lemma 2.5 together with parabolic regularity implies

Proposition 3.5. The mapping $\mathcal{F}_{3}$ is $C^{1}$ and compact from $\left[\underline{c_{0}} / 2,2 \bar{c}_{0}\right] \times X_{r} \times X_{r}$ into $X_{r}$.

Finally, we define the mapping

$$
\mathcal{F}_{1}(c, Y, W)=c-\left(W(0,0)-Y(0,0)-\theta_{i}\right)
$$

Clearly, $\mathcal{F}_{1}$ is compact. 
We are now ready to state the fixed point problem. Let $C>0$ be such that if ( $c, T, Y, S$ ) solves (3.4)-3.7), then the corresponding functions $u$ and $w$ defined by (3.12) are-due to all the estimates of Section 2-in the open ball of $X_{r}$ with centre 0 and radius $C$, which we denote by $B_{r}(0, C)$. Now, define the open subset of $\mathbb{R} \times X_{r} \times X_{r}$ and the mapping $\mathcal{F}$ from $\Omega$ to $\mathbb{R} \times X_{r} \times X_{r}$ by

$$
\Omega=\left(\underline{c_{0}} / 2,2 \bar{c}_{0}\right) \times B_{r}(0, C) \times B_{r}(0, C), \quad \mathcal{F}=\left(\mathcal{F}_{1}, \mathcal{F}_{2}, \mathcal{F}_{3}\right) .
$$

Clearly, $\mathcal{F}$ is compact from $\Omega$ to $X_{r} \times X_{r} \times \mathbb{R}$. Moreover, a fixed point of $\mathcal{F}$ cannot be on $\partial \Omega$.

\subsection{The homotopy}

To do the homotopy, we simply perturb the values at $-\infty$. Without loss of generality, we assume that $\theta_{i}<1 / 2$. We then replace $Y_{u}$ as the left limit of $Y$ by $\tau Y_{u}+1-\tau$, and $S_{u}$ by $\tau S_{u}$-that is, we replace $T_{b}\left(Y_{u}, S_{u}\right)$ by $T_{b}\left(\tau Y_{u}+1-\tau, \tau S_{u}\right)$. We note that, for $\tau \in[0,1]$, the estimates of Section 2 apply to these new conditions at $-\infty$; we let $\mathcal{F}^{\tau}$ be the corresponding mappings defined in the preceding section. Clearly, $(\tau, c, u, w) \mapsto$ $\mathcal{F}^{\tau}(c, u, w)$ is $C^{1}$ and compact. Also, any fixed point of $\mathcal{F}^{\tau}$ is inside $\Omega$ according to the a priori estimates. We may therefore define $\operatorname{deg}\left(I-\mathcal{F}^{\tau}, \Omega, 0\right)$; it is constant with respect to $\tau$. This triggers the last step of

Proof of Theorem 1.1. It suffices to prove that $\operatorname{deg}\left(I-\mathcal{F}^{\tau}, \Omega, 0\right) \neq 0$. However, for $\tau=0$, we have the usual thermo-diffusive system with the Lewis number equal to one: there are no droplets. Hence, a fixed point of $I-\mathcal{F}^{\tau}$ is such that the corresponding function $W$ is exactly equal to 1 , and the corresponding function $Y$ is a solution of

$$
Y_{t}-Y_{x x}+c Y_{x}=-Y f(1-Y):=g(Y) .
$$

The only time-periodic solution of (3.17) such that $1-Y$ is equal to $\theta_{i}$ at $(0,0)$ and goes to 0 at $+\infty$ is the $1 \mathrm{D}$ wave that we call $Y_{0}$ with the speed called $c_{0}$. Let us quickly prove that $I-\partial_{c, u, w} \mathcal{F}^{0}$ at the wave is an isomorphism of $\mathbb{R} \times X_{r} \times X_{r}$; notice that, because of what precedes, $\partial_{c, u, w} \mathcal{F}^{\tau}$ is a compact operator on $\mathbb{R} \times X_{r} \times X_{r}$. Hence it suffices to prove that $I-\partial_{c, u, w} \mathcal{F}^{0}$ has a zero null space. By the definition of $\mathcal{F}^{\tau}$, it suffices to solve the following equation, with unknowns $(\tilde{c}, \tilde{u}, \tilde{w})$ :

$$
\left\{\begin{array}{l}
\tilde{u}(0,0)=0, \\
\left(\partial_{t}-\partial_{x x}+c_{0} \partial_{x}-g^{\prime}\left(Y_{0}\right)\right) \tilde{u}=0, \quad u(t+1, \cdot)=u(t, \cdot) . \\
\tilde{w}=0,
\end{array}\right.
$$

From [9. Chap. 5], the operator

$$
L_{0}=-\frac{d^{2}}{d x^{2}}+c_{0} \frac{d}{d x}-g^{\prime}\left(Y_{0}\right),
$$

with domain in $X_{r}$, is nondegenerate, in the sense that the geometric and algebraic multiplicity of the eigenvalue 0 is 1 , with associated eigenfunction $Y_{0}^{\prime}$. Consequently, the 
second line of 3.18 implies that $u(t, x)$ is proportional to $Y_{0}^{\prime}$, and the first equation in turn implies that $u \equiv 0$.

To summarize, $\mathcal{F}^{0}$ has a unique zero in $\Omega$, and $\partial_{c, u, w} \mathcal{F}^{0}$ is an isomorphism of $\mathbb{R} \times$ $X_{r} \times X_{r}$, which implies (see [11]) that $\operatorname{deg}\left(I-\mathcal{F}^{\tau}, \Omega, 0\right)$ is nonzero. This ensures the existence of a wave solution to 2.1]-2.3 as soon as the evaporation temperature $\theta_{v}$ is positive.

It remains to send $\theta_{v}$ to 0 . However, all the bounds that are proved in Section 2 are uniform with respect to $\theta_{v}$, as soon as $\theta_{i}$ is fixed-in fact, $\theta_{v}>0$ was only required to obtain some compactness for $W$. The passage to the limit $\theta_{v} \rightarrow 0$ is therefore standard; see for instance [6] for the details.

\section{The KPP limit}

As in [6], the strategy that we shall use here for obtaining the wave of lower velocity is to send the ignition temperature to 0 . Our main problem is that the bounds devised so far are not uniform with respect to the ignition temperature. On the other hand, what we are now aiming at is the existence of waves when both ignition and vaporization temperatures are zero. This leaves us some freedom for the approximating sequences, and we will use one that will generate a painless estimate for $Y$-something that we had to work for in Section 2. This in turn will allow us a (less easy) estimate for the enthalpy. Also, we will in a first approximation keep the mass fraction of the unburnt gases nonzero; this will give us a free lower estimate, for in this case, part 1 of the proof of Proposition 2.1 applies. All this is summed up in the following

Proposition 4.1. Let $\left(f_{\theta}\right)_{\theta>0}$ and $\left(\varphi_{\theta}\right)_{\theta>0}$ be two sequences of Lipschitz functions, defined for small $\theta>0$ and having $\theta$ as ignition (resp. vaporization) temperatures. Assume moreover the ratio $\varphi_{\theta} / f_{\theta}$ to be uniformly bounded from above, and bounded away from 0 . Consider $Y_{u}>0$ and a positive, smooth, 1-periodic function $S_{u}(x)$. Then there exists a family of solutions $\left(c_{\theta}, T_{\theta}, Y_{\theta}, S_{\theta}\right)$ to the problem 2.1)-2.3. Moreover, the following properties hold:

- the sequence $\left(c_{\theta}\right)$ is bounded away from 0 ,

- if $W_{\theta}$ is the enthalpy, then the sequence $\left\|W_{\theta}\right\|_{\infty}$ is bounded,

- the exponential estimates of Section 2.3 hold uniformly with respect to $\theta$.

Proof. It is clear that, given the considerations of Sections 2 and 3 , a solution $\left(c_{\theta}, T_{\theta}, Y_{\theta}, S_{\theta}\right)$ to the problem 2.1]-2.3 exists as soon as the estimates stated in the proposition hold, and the proof reduces to proving these estimates. In what follows, we consider a solution $(c, T, Y, S)$ to 2.1)-2.3); we have deleted (and will continue to do so in the course of the proof) the subscript ${ }_{\theta}$ in order to alleviate the notations.

1. Upper bound for $Y$. Break $Y(t, x)$ into $Y_{1}(t, x)+Y_{2}(t, x)$ where

$$
\left\{\begin{array}{l}
\left(\partial_{t}-\partial_{x x}+c \partial_{x}+f(T)\right) Y_{1}=0 \quad(t>0, x \in \mathbb{R}) \\
Y_{1}(0, x)=Y(0, x)
\end{array}\right.
$$


and

$$
\left\{\begin{array}{l}
\left(\partial_{t}-\partial_{x x}+c \partial_{x}+f(T)\right) Y_{2}=\varphi(T) S \quad(t>0, x \in \mathbb{R}) \\
Y_{2}(0, x)=0
\end{array}\right.
$$

By an elementary computation for the advection-diffusion equation we have

$$
\limsup _{t \rightarrow+\infty} Y_{1}(t, x) \leq Y_{u}
$$

moreover, if the function $(s, x) \mapsto Y_{2}(s, x)$ has a maximum point $\left(t_{0}, x_{0}\right)$, then

$$
Y_{2}\left(t_{0}, x_{0}\right) \leq \frac{\varphi\left(T\left(t_{0}, x_{0}\right)\right.}{f\left(T\left(t_{0}, x_{0}\right)\right.} S\left(t_{0}, x_{0}\right) \leq C\left\|S_{u}\right\|_{\infty},
$$

as the ratio $\phi / f$ is uniformly bounded by assumption. If there is no maximum, we may always consider a maximizing sequence $\left(t_{n}, x_{n}\right)$, consider the suitably translated sequence $Y\left(t+t_{n}, x+x_{n}\right)$ and send $n$ to $+\infty$, to get the same estimate. In any case, this bounds $Y$ from above.

2. Lower bound for $c$. Similar to part 1 of the proof of Proposition 2.1.

In the next two steps we revert (for convenience, and without change of notation) to the original reference frame; thus the functions $(T, Y, S)$ satisfy $1.1-1.3$. Of course, the benefit of the estimates of the previous two steps is kept.

3. $L^{\infty}$ bound for $T_{t}$ and $T_{x x}$. We start from the Duhamel formula for $W(t, x)$ :

$$
W(t, x)=e^{t \partial_{x x}} W(0, x)+\int_{0}^{t} \int_{\mathbb{R}} \frac{e^{-(x-y)^{2} / 4(t-s)}}{\sqrt{4 \pi(t-s)}}\left(-\partial_{s} S^{3 / 2}\right) d s .
$$

The free term $e^{t \partial_{x x}} W(0, x)$ has the eventual bound $T_{b}\left(Y_{u}, S_{u}\right) / 2$ and is of no concern. The remaining term, which we denote by $W_{1}(t, x)$, is broken into

$$
W_{1}(t, x)=\int_{0}^{t-1}+\int_{t-1}^{t}=: W_{11}+W_{12},
$$

which we study separately.

[i] Because $\varphi(T)$ and $S$ are both bounded (see (1.2), we immediately infer from the parabolic regularity that, for every $p \in(1,+\infty)$, there is $C_{p}>0$ such that, for every bounded interval $I$ of length $1 / 2$, and for every $(t, x) \in(2,+\infty) \times \mathbb{R}$, we have

$$
\left\|\partial_{t} W_{12}\right\|_{L^{p}\left((t, x)+I^{2}\right)}+\left\|\partial_{x x} W_{12}\right\|_{L^{p}\left((t, x)+I^{2}\right)} \leq C_{p} .
$$

Indeed, $W_{12}$ solves

$$
\begin{aligned}
& \left(\partial_{s}-\partial_{x x}\right) W_{12}=-\partial_{s} S^{3 / 2}=\frac{3}{2} \varphi(T) S^{3 / 2} \quad \text { for }(s, x) \in(t-1, t) \times \mathbb{R}, \\
& W_{12}(t-1, x)=0 .
\end{aligned}
$$


[ii] The term $W_{11}$ has the expression

$$
\begin{aligned}
W_{11}(t, x)= & \int_{\mathbb{R}}\left(\frac{e^{-(x-y)^{2} / 4}}{\sqrt{4 \pi}} S(t-1, y)^{3 / 2}-\frac{e^{-(x-y)^{2} / 4 t}}{\sqrt{4 \pi t}} S(0, y)^{3 / 2}\right) d y \\
& +\int_{0}^{t-1} \int_{\mathbb{R}} \frac{1}{t-s}\left(1-\frac{(x-y)^{2}}{2(t-s)}\right) \frac{e^{-(x-y)^{2} / 4(t-s)}}{\sqrt{4 \pi(t-s)}} S(s, y)^{3 / 2} d s d y \\
=: & W_{111}(t, x)+W_{112}(t, x) .
\end{aligned}
$$

The term $\partial_{t} W_{111}$ is uniformly bounded, just because $S_{t}$ is uniformly bounded. As for $W_{112}$ there is a polynomial $P(X)$ (easily explicitly computed, but whose expression is of no use to us) such that

$$
\left|\partial_{t} W_{112}(t, x)\right| \leq \int_{0}^{t-1} \frac{1}{(t-s)^{2}} P\left(\frac{(x-y)^{2}}{t-s}\right) e^{-(x-y)^{2} / 4(t-s)} S(s, y)^{3 / 2} d s d y .
$$

This bounds $\partial_{t} W_{112}$.

Now, remembering 4.1) and using the equation for $W$ and the boundedness of its right side, we conclude that the outcome of the two paragraphs [i] and [ii] is

$$
\left\|\partial_{t} W\right\|_{L^{p}\left((t, x)+I^{2}\right)}+\left\|\partial_{x x} W\right\|_{L^{p}\left((t, x)+I^{2}\right)} \leq C_{p}
$$

for all $p \in(1,+\infty)$. This is not quite enough; we would in fact wish to bound $\partial_{t} W$ and $\partial_{x x} W$ in some Hölder space. However we are now in a relatively good situation, and we may argue as follows: first, the boundedness of the coefficients of the equation for $Y$, as well as the boundedness of $Y$, imply a bound for $Y$ of the type (4.3). This in turn implies a similar bound for $T$, because $W=T-Y$. Consequently, because $\varphi(T)$ and $f(T)$ are bounded together with their derivatives, there is $\alpha \in(0,1)$ such that

$$
\|f(T)\|_{C^{\alpha, \alpha / 2}}+\|\varphi(T)\|_{C^{\alpha, \alpha / 2}} \leq C .
$$

If we now set $u(t, x)=T_{t}(t, x)$ we have, from the previous considerations,

$$
u_{t}-u_{x x}-f^{\prime}(T) Y u=Y_{t} f(T)
$$

the coefficients and RHS of the above equation are bounded in $C^{\alpha, \alpha / 2}$. Moreover, because of the $L_{\text {loc }}^{p}$ bound for $u$, there is $t_{0} \in(0,1)$ such that $\left\|u\left(t_{0}, \cdot\right)\right\|_{L^{p}(I)}$ is bounded uniformly on all intervals $I$ of length 1. Parabolic regularity implies a Hölder bound for $u_{t}$, which is enough to infer a Hölder bound for $W_{t}$. Hence the outcome of this step is

$$
\left\|T_{t}\right\|_{\infty}+\left\|T_{x x}\right\|_{\infty} \leq C .
$$

4. $L^{\infty}$ bound for $W$. If $C$ is the bound of (4.4), and $\underline{c_{0}}$ a lower bound for $c$, consider $K>0$ large enough so that

$$
K \geq 1+2 \frac{C+3 \sqrt{C K}}{\underline{c_{0}}}
$$


let $x_{0}$ be the smallest point $x$ for which there is $t \in \mathbb{R}_{+}$such that $T(t, x)=K$; without loss of generality we may assume that $(t, x)=(0,0)$. Hence we have $T(k,-c k)=K$; recall that at the moment we are working in the original variables. Now, by interpolation, using 4.5 and (4.4) we have $T(t,-c t) \geq 1$ for all $t \in \mathbb{R}$. Letting $t \rightarrow+\infty$ yields

$$
T(t, x) \geq \min \left(1, T_{b}\left(Y_{u}, S_{u}\right)\right),
$$

for $x>-c t$, which in turn implies that $\varphi(T)$ is bounded away from 0 by a constant $\underline{\varphi}$ independent of $\theta$. Consequently, for $t>0$ and $x>-c t$ we have

$$
S(t, x) \leq\left\|S_{u}\right\|_{\infty} \exp \{-\underline{\varphi}(t-x / c)\},
$$

and $u(t, x):=W(t, x-c t)$ satisfies, for $x>0$,

$$
\left|u_{t}-u_{x x}+c u_{x}\right| \leq C\left\|S_{u}\right\|_{\infty} e^{-\underline{\varphi} t} .
$$

Due to the upper bound for $Y$, this is bounded for $x=0$ and $x=+\infty$. Letting $t \rightarrow+\infty$ yields a uniform bound for $u$ and $W$.

Once the $L^{\infty}$ bound for $W$ is obtained, the upper bound for $c$ and the exponential bounds follow as in Section 2.2

Proposition 4.1 readily implies Theorem 1.2. Indeed, one only has to consider a sequence of approximating solutions $\left(c_{\theta}, T_{\theta}, Y_{\theta}, S_{\theta}\right)$. The uniform $L^{2}$ estimate plus the lower bound on $c_{\theta}$ ensure that the limiting triple $(T, Y, S)$ converges to the right limit at $\pm \infty$; the details are as in [5].

\section{Existence of waves with higher velocities}

A first trivial observation to support this fact—which also has the merit of clearly pointing out where the $Y_{u}>0$ assumption is needed-is the following: any solution $(T, Y, S)(t, x)$ of the Cauchy problem for $1.1-1.3$ has $W(t, x) \geq Y_{u} / 2$ as soon as we wait long enough; consequently, if $f(T)=T$ we have

$$
W-T \geq\left(Y_{u} / 2-T\right) T
$$

for $t>0$ large. The RHS of the above inequality is, once again, a KPP term, which generates travelling waves connecting 0 to $Y_{u} / 2$ with any speed larger than $\sqrt{Y_{u} / 2}$. We therefore may have arbitrarily high burning rates, in the sense of [8], hence arbitrary large propagation velocities might be expected.

Let us now try to give some substance to this observation. To do so, we will be guided by the following toy problem:

$$
u_{t}-u_{x x}=\left(Y_{u}-u\right) u=: f_{0}(u), \quad u(t,-\infty)=0, u(t,+\infty)=Y_{u} .
$$

For every $K>0$ and $c>2 \sqrt{K}$, let us define the two quantities $r_{-}(c, K)<r_{+}(c, K)$ by

$$
r_{ \pm}(c, K)=\frac{c \pm \sqrt{c^{2}-4 K}}{2} .
$$


For every $c>2 \sqrt{Y} u$ a travelling wave solution $\phi_{c}(x)$ of 5.1 that moves with the speed $c$ exists and decays at $-\infty$ according to the minimal rate [13], i.e. for every $r<c$ there exists $k_{r}>0$ such that

$$
\phi_{c}(x)=k_{r} e^{r_{-}\left(c, Y_{u}\right) x}+O\left(e^{r x}\right) .
$$

Moreover, still for every $r \in(0, c)$, define the weighted space

$$
B_{r}=\left\{u(x) \in B U C(\mathbb{R}):\left(1+e^{-r x}\right) u \in B U C(\mathbb{R})\right\} ;
$$

then the operator $L$, with a suitable domain in $B_{r}$, defined as

$$
L=-\frac{d^{2}}{d x^{2}}+c \frac{d}{d x}-f_{0}^{\prime}\left(\phi_{c}\right)
$$

is an isomorphism from its domain into $B_{r}$; see once again [13].

For the construction of waves with higher velocities, we are going to use a degree argument similar in spirit to the one of Section 3, apart from the fact that the velocity is now prescribed. Note that, once the velocity is prescribed, $L^{\infty}$ bounds for $T, W$ and $S$ can be obtained by arguing as in, for instance, the proof of Proposition 4.1. We will use these bounds freely, without writing them in the form of a theorem.

\subsection{Direct lower bound on the velocity}

Theorem 1.2 does not by any means imply Theorem 1.3 Indeed, it yields a pulsating wave solution whose velocity is bounded in terms of the data; however, it does not say that all pulsating wave solutions to (1.1)- 1.3 satisfy this estimate. Of course, it also says nothing about the boundedness or unboundedness of the velocity spectrum.

The first task in proving Theorem 1.3 is to prove a direct lower bound on the wave speeds. We start with a qualitative property of the temperature analogous to, but weaker than, the lap number decay principle, which will be useful to us in what follows.

Proposition 5.1. If $(c, T, Y, S)$ is a solution to (2.1)-(2.3) then, for all $t \in \mathbb{R}$, the function $x \mapsto T(t, x)$ is nondecreasing on the set where it is below $T_{b}\left(Y_{u}, S_{u}\right)$.

Proof. Assume the contrary. Then there is a value $l \in\left(0, T_{b}\left(Y_{u}, S_{u}\right)\right)$ that is, at some time $t_{0}$, taken twice by $x \mapsto T\left(t_{0}, x\right)$. By Sard's theorem, we may assume this value to be noncritical for the function $T$. The level set $\{T(t, x)=l\}$ consists, therefore, of a finite set of ordered, nonintersecting smooth curves $\left\{t, y_{i}(t)\right\}$ in space-time. Take any $i$ such that $T(t, x)<l$ if $x \in\left(y_{i}(t), y_{i+1}(t)\right)$. This defines an open subset $\Omega$ in space-time in which, by periodicity of $T$, a minimum is attained. However, $T$ is a supersolution to an advection-diffusion equation, which contradicts the strong maximum principle.

Proof of Theorem 1.3. Step 1: a lower bound on velocities. It shares many common points with the proof of Proposition 2.1. apart from the fact that we may not get an upper bound for the temperature in the unburnt region-for the simple reason that there is no unburnt region, as there is no ignition temperature. We argue by contradiction, that is, assume the 
existence of a sequence $c_{n} \rightarrow 0$-that we immediately relabel simply $c$-giving rise to pulsating wave solutions. We use once again the parabolic scaling

$$
\tau=c^{2} t, \quad \xi=c x .
$$

Consider $A>0$ large; the size of $A$ (independent of $c$ ) will be made precise later. From Proposition 5.1 there exists a possibly discontinuous, but at least lower semicontinuous function $\xi_{A}(\tau)$ such that

$$
T(\tau, \xi) \geq A c^{2} \quad \text { if and only if } \xi \geq \xi_{A}(\tau) .
$$

We may assume that the minimum of $\xi_{A}$ is 0 . For $\xi \geq 0$ we have

$$
\left(\partial_{\tau}-\partial_{\xi \xi}+\partial_{\xi}\right) T \geq 0 .
$$

Arguing as in the proof of Proposition 2.1 we infer the existence of a $\delta>0$, independent of $A$ and $c$, such that

$$
\forall(\tau, \xi) \in \mathbb{R} \times \mathbb{R}_{+}, \quad T(\tau, \xi) \geq \delta A c^{2} .
$$

Now, remember that 5.6 also holds for $\xi \leq 0$; as a consequence we have

$$
\forall(\tau, \xi) \in \mathbb{R} \times \mathbb{R}_{-}, \quad T(\tau, \xi) \geq \delta A c^{2} e^{\xi} .
$$

In particular, we have

$$
T(\tau,-2) \geq \delta A c^{2} / e^{2}
$$

Turn now to $S(\tau, \xi)$; recall the equation

$$
\left(\partial_{\tau}+\partial_{\xi}\right) S=-\varphi(T) S / c^{2},
$$

and the fact that $C_{1} T \leq \varphi(T) \leq C_{2} T$; together they imply

$$
\forall(t, \xi) \in \mathbb{R} \times[-2,+\infty), \quad S(\tau, \xi) \leq\left\|S_{u}\right\|_{\infty} \exp \left(-\frac{C \delta A}{e^{2}}(\xi+2)\right) .
$$

Finally, turn to the function $W(\tau, \xi)$. The time period of the wave in the rescaled coordinates is $c$; however, we may also consider the wave as being $\tau_{0}=N c$-periodic, with $N=[1 / c]$. Note that when $c$ is small, which is the case here, $\tau_{0}$ is a number in the interval $[1 / 2,1]$. This observation will be useful to us when we wish to apply parabolic regularity. Let $w_{0}(\xi)$ be the zeroth Fourier mode of $W$, that is, its average over a time period; we have, for $-2 \leq \xi \leq 0$,

$$
\begin{aligned}
w_{0}(\xi) & =Y_{u}+\left\langle S_{u}^{3 / 2}\right\rangle-\int_{\xi}^{+\infty} e^{\xi-\zeta}\left\langle S^{3 / 2}\right\rangle(\zeta) d \zeta \\
& \geq\left\langle S_{u}^{3 / 2}\right\rangle-\left\|S_{u}\right\|_{\infty}^{3 / 2} /(1+C \delta A) \quad \text { by } 5.10 \\
& \geq\left\langle S_{u}^{3 / 2}\right\rangle / 2 \quad \text { as soon as } A \text { is large enough. }
\end{aligned}
$$


Consider now $A$ to be chosen so that the last inequality of 5.11 holds; since $\varphi(T)$ is uniformly bounded for $-2 \leq \xi \leq 0$, we have

$$
\left(\partial_{\tau}-\partial_{\xi \xi}+\partial_{\xi}\right) W=\frac{3}{2 c^{2}} S^{3 / 2} \varphi(T)=O(1) .
$$

By parabolic regularity, and also by letting $\tau \rightarrow+\infty$, so that we only keep the effect of the right side, for all $p>1$, there is $C_{p}>0$ independent of $c$ such that

$$
\left\|W_{\tau}\right\|_{L^{p}([0,1] \times[-2,0])}+\left\|W_{\xi \xi}\right\|_{L^{p}([0,1] \times[-2,0])} \leq C_{p} .
$$

Take $p$ large enough so that the above estimate implies a $C^{\alpha, \alpha / 2}$ estimate for some $\alpha \in$ $(0,1)$. This, combined with 5.11, and the fact that $W$ is $c$-periodic in $\tau$, and $c$ is a small number, implies

$$
\forall \tau \in \mathbb{R}, \quad W(\tau,-1) \geq\left\langle S_{u}^{3 / 2}\right\rangle / 3 .
$$

Next, we recall that $T(\tau, \xi) \leq A c^{2}$ for all $\tau$ and $\xi \leq 0$. Consider now a small number $\theta$ and a smooth function $g(T)$ having $\theta$ as an ignition temperature, and such that $g(T) \leq T$. We therefore have $A c^{2} \leq \theta$ if $c$ is small enough; hence, for our pulsating wave we have

$$
(W-T) T \geq\left(\left\langle S_{u}^{3 / 2}\right\rangle / 3-T\right) g(T) \quad \text { on } \mathbb{R}^{2} .
$$

Indeed, 5.13 holds for $\xi>-1$ because of 5.12, while for $\xi \leq-1$ the right side of (5.13) vanishes because $T$ is below the ignition temperature for $g(T)$. The velocity of the pulsating wave is therefore larger than the velocity $c_{\theta}^{0}$ of the travelling wave solution of

$$
u_{t}-u_{x x}=\left(\left\langle S_{u}^{3 / 2}\right\rangle / 3-u\right) g(u), \quad u(t,-\infty)=0, u(t,+\infty)=\left\langle S_{u}^{3 / 2}\right\rangle / 3
$$

When $\theta>0$ is small enough, we have $c>c_{\theta}^{0} \sim 2 \sqrt{\left(\left\langle S_{u}^{3 / 2}\right\rangle / 3\right) f^{\prime}(0)}$, the KPP velocity. This contradicts the smallness of $c$.

\subsection{Uniform decay bounds and homotopy}

The general idea is the following: perform a homotopy from problem (1.1)- 1.3 to problem (5.1). The deformation is done through the droplet distribution at $-\infty$; namely we go from $S(t, x)=S_{u}(x)$ at $-\infty$ to $S(t, x)=0$ at $-\infty$. This means that we simply forget, in the end, the effect of the droplets, and this is understandable: combustion will occur in this situation whether or not droplets are present in the picture.

What we will need to complete the degree argument is not only the classical $L^{2}$ bound on $T$ at $+\infty$, but a uniform control of $T$ in the $X_{r}$ norm, for some $r \in\left(r_{-}\left(c, Y_{u}\right), c\right)$. This will allow us to reduce the issue to the problem of finding a fixed point of an operator which is a sum of a contracting and a compact one. 
Proposition 5.2. There exist $K>0$ and $\delta_{0}>0$ satisfying $r_{-}\left(c, Y_{u}\right)+\delta_{0}<r_{+}\left(c, Y_{u}\right)$, depending only on the data, such that if $(T, W, S)$ is a pulsating wave solution to (1.1) (1.3) with velocity $c \geq K$, and

$$
T(t, x)-e^{r_{-}\left(c, Y_{u}\right) x}=O\left(e^{-\left(r_{-}\left(c, Y_{u}\right)+\delta_{0}\right) x}\right) \quad \text { as } x \rightarrow-\infty,
$$

then there exists $C>0$, also depending only on the data, such that

$$
\left|T(t, x)-e^{r_{-}\left(c, Y_{u}\right) x}\right| \leq C e^{\left(r_{-}\left(c, Y_{u}\right)+\delta_{0}\right) x} \quad \text { for }(t, x) \in \mathbb{R}^{2} .
$$

Proof. The proof of this proposition is really a stability argument; instead of comparing directly $T(t, x)$ to $e^{r_{-}\left(c, Y_{u}\right) x}$ we will compare it to the only (KPP) wave $\phi_{0}(x)$ of $[5.1$, satisfying

$$
\phi_{0}(x) \sim e^{r_{-}\left(c, Y_{u}\right) x} \quad \text { as } x \rightarrow-\infty .
$$

1. We claim that, if $T(t, x) \sim \phi_{0}(x)$ for $x \rightarrow-\infty$, then $T(t, x) \geq \phi_{0}(x)$. Indeed, $W(t, x) \geq Y_{u}$, implying

$$
\left(\partial_{t}-\partial_{x x}+c \partial_{x}\right) T \geq\left(Y_{u}-T\right) T, \quad T(t,+\infty) \geq Y_{u} .
$$

Let $u(t, x)$ be the only solution of 5.1 with the initial datum $T(0, x)$. Because of 5.14 we have (see [14])

$$
\lim _{t \rightarrow+\infty} u(t, x)=\phi_{0}(x) .
$$

This, together with 5.17), proves the claim.

2. Consider $\delta>0$ small, to be chosen later. Assume the function $T(t, x)$ has been translated in time and space such that

$$
T(0,0)=\min _{(t, x) \in \mathbb{R} \times \mathbb{R}_{+}} T(t, x)=\delta .
$$

This implies, through Step 1, that the corresponding $\phi_{0}(x) \leq \delta$ for $x \leq 0$. It also follows that $\varphi(T)=f(T)=T$ for $\xi \leq 0$. Now, for all $\xi \in \mathbb{R}_{-}$, denote by $w(t, \xi)$ the function $W(t, \xi)$ and decompose $W(t, x)$, for $t \in \mathbb{R}, x \leq \xi$, as $W[T]+W^{0}$, where both functions $W[T]$ and $W^{0}$ are $1 / c$-periodic in $t$, and where

$$
\begin{array}{rlrl}
\left(\partial_{t}-\partial_{x x}+c \partial_{x}\right) W[T] & =\frac{3}{2} S^{3 / 2} T, & & W[T](t, \xi)=0, \\
\left(\partial_{t}-\partial_{x x}+c \partial_{x}\right) W^{0} & =0, & W^{0}(t, \xi)=w(t, \xi) .
\end{array}
$$

We recall the existence of $C>0$ such that

$$
0 \leq W^{0}(t, x)-Y_{u} \leq C e^{c(x-\xi)},
$$

simply because the right side of 5.20 ) is a supersolution to the equation 5.19 ) for $W^{0}$, and because of the $L^{\infty}$ bounds for $W$. Now, set

$$
r_{\delta}=r\left(c, Y_{u}\right)+\delta, \quad v(t, x, \xi)=e^{-r_{\delta}(x-\xi)}\left(T(t, x)-\phi_{0}(x)\right) ;
$$


the real number $\delta$ is not chosen yet—simply remember that it will be small. The function $v(t, x, \xi)$ solves, for $(t, x) \in \mathbb{R} \times(-\infty, \xi)$,

$$
\begin{aligned}
v_{t}-v_{x x}+(c- & \left.2 r_{\delta}\right) v_{x}+\left(c r_{\delta}-r_{\delta}^{2}-Y_{u}\right) v \\
& =-\left(\phi_{0}+T\right) v+\left(W^{0}-Y_{u}\right) e^{-r_{\delta}(x-\xi)} T+e^{-r_{\delta}(x-\xi)} W[T] T \\
& \leq e^{-r_{\delta}(x-\xi)}\left(C \delta e^{\left(c-r_{\delta}\right)(x-\xi)}+W[T] T\right) .
\end{aligned}
$$

In the last inequality of (5.22), we have used the positivity of $v$ to drop the first term on the second line, while in the second term we used the decay of $T \sim \phi_{0}(x)$, and 5.20). Set

$$
V_{\delta}(t, \xi)=\|v(t, \cdot, \xi)\|_{L^{\infty}((-\infty, \xi))}
$$

recall that an eventual supersolution for $W[T]$ is the function $\bar{W}(t, x)$ solving

$$
-\bar{W}^{\prime \prime}+c \bar{W}^{\prime}=\frac{3}{2}\left\|S_{u}\right\|_{\infty}^{3 / 2}\left(\phi_{0}+e^{r_{\delta}(x-\xi)} V_{\delta}(t, \xi)\right) .
$$

We invoke the following three facts:

(i) $r<c$,

(ii) formula 2.16 holds and gives an expression of $\bar{W}$,

(iii) we have $\phi_{0}(x) \leq C \delta e^{r_{-}\left(c, Y_{u}\right)(x-\xi)}$ - see Step 1 of this proof. The constant $C$ is independent of $\delta>0$.

Points (i) to (iii) above imply, after a computation, the following bound for $W[T] T$ on $\mathbb{R} \times(-\infty, \xi)$ :

$$
0 \leq e^{-r_{\delta}(x-\xi)} W[T] T \leq C\left(e^{\left(r_{-}\left(c, Y_{u}\right)-\delta\right)(x-\xi)}+e^{r_{\delta}(x-\xi)} V(t, \xi)^{2}\right) \delta^{2} .
$$

Now, we start shrinking $\delta$. First, we require the amount $c r_{\delta}-r_{\delta}^{2}-Y_{u}$ to be positive; from (5.22)-(5.24), the normalization condition (5.18) and the maximum principle, we have

$$
V_{\delta}(t, \xi) \leq \delta+e^{-\left(c r_{\delta}-r_{\delta}^{2}-Y_{u}\right) t} V_{0}+C \delta^{2}\left(1+\int_{0}^{t} e^{\left(c r_{\delta}-r_{\delta}^{2}-Y_{u}\right)(t-s)} V_{\delta}^{2}(s, \xi) d s\right) .
$$

Then we ask $\delta$ to be small enough so that the equation $C \delta X^{2}-X+C \delta$ has two positive roots: one that is $O(\delta)$, the other one that is $O(1 / \delta)$. Fix such a $\delta$, and call it $\delta_{0}$.

Letting $t \rightarrow+\infty$ and using the $1 / c$-periodicity of $V_{\delta}(t, \xi)$ we get, for all $\delta \leq \delta_{0}$, and for a constant $C$ once again independent of $\delta$,

$$
\begin{aligned}
\left\|V_{\delta}(\cdot, \xi)\right\|_{\infty} & \leq C\left(\delta+\delta^{2}+\frac{\delta^{2}}{c r_{\delta}-r_{\delta}^{2}-Y_{u}}\left\|V_{\delta}(\cdot, \xi)\right\|_{\infty}^{2}\right) \\
& \leq C \delta\left(1+\left\|V_{\delta}(\cdot, \xi)\right\|_{\infty}^{2}\right) .
\end{aligned}
$$

This implies that either $V_{\delta}(t, \xi)=O(\delta)$, or $V_{\delta}(t, \xi)=O(1 / \delta)$. The first solution prevails for large negative $\xi$ and small $\delta$; so by continuity, $V_{\delta_{0}}(t, 0)=O\left(\delta_{0}\right)$. This proves our proposition. 
The fact that $T \geq \phi_{0}$ readily implies the exponential convergence of $S$ and $W$ to their limits at $-\infty$.

Lemma 5.3. There are $\rho_{0}>0$ and $C>0$, depending only on the data, such that

$$
\forall(t, x) \in \mathbb{R} \times \mathbb{R}_{-}, \quad 0 \leq S_{u}(x)-S(t, x) \leq C e^{\rho_{0} x}, \quad 0 \leq W(t, x)-Y_{u} \leq C e^{\rho_{0} x} .
$$

The proof is at this stage routine and is omitted. The last ingredient that we need for the homotopy is a quantitative, uniform decay to the right for the functions $T-Y_{u}-\left\langle S_{u}^{3 / 2}\right\rangle$, $W-Y_{u}-\left\langle S_{u}^{3 / 2}\right\rangle$. This is provided by

Proposition 5.4. There is $\rho_{0}>0$ such that if $T(t, x)$ satisfies the assumptions of Proposition 5.2, then, for some $C>0$ depending only on the data,

$$
\forall(t, x) \in \mathbb{R} \times \mathbb{R}_{+}, \quad\left|(T(t, x), W(t, x))-\left(T_{b}\left(Y_{u}, S_{u}\right), T_{b}\left(Y_{u}, S_{u}\right)\right)\right| \leq C e^{-\rho_{0} x} .
$$

Proof. Come back to a pulsating wave that satisfies the normalization condition (5.18). Arguing as in Proposition 2.1 we have $T(t, x) \geq C \delta_{0}$ for $x \geq 0$; on the other hand, we have

$$
S_{t}+c S_{x}+S \varphi(T)=0
$$

together these facts imply a uniform exponential decay for $S$. Then $Y(t, x)$ satisfies

$$
Y_{t}+c Y_{x}-Y_{x x}+f(T) Y=\frac{3}{2} S^{3 / 2} \varphi(T) ;
$$

using the lower bound for $T$ on $\mathbb{R} \times \mathbb{R}_{+}$, and the exponential bound for $S$ on the same set, implies the exponential bound for $Y$. The function $W$ is then treated as in Proposition 2.4.

Proof of Theorem 1.3 (end). Let us pick $c$ satisfying the assumptions of Proposition 5.2 Let $\gamma$ be a smooth, nonnegative function, equal to 1 on $\mathbb{R}_{-}$and 0 on $[1,+\infty)$. Let $\delta_{0}$ satisfy the conclusions of Proposition 5.2, and set once and for all

$$
r=r\left(c, Y_{u}\right)+\delta_{0}
$$

The fixed point setting that we are going to devise here is simpler than in Section 3, because $c$ is not to be looked for. Let us define the space $X_{r, \delta_{0}}$ as

$$
X_{r, \delta_{0}}=\left\{u \in C_{\mathrm{per}}^{\alpha, \alpha / 2}:\left(e^{-r x}\left(u-\phi_{0}\right), e^{\delta_{0} x}\left(u-Y_{u}-\left\langle S_{u}^{3 / 2}\right\rangle\right)\right) \in C_{\mathrm{per}}^{\alpha, \alpha / 2}\right\} .
$$

Here the space $C_{\text {per }}^{\alpha, \alpha / 2}$ means the classical Hölder functions which are additionally $1 / c$ periodic in $t$. Now, for $T \in X_{r}$, let $\mathcal{F}_{1}[T]$ be defined as (3.10), i.e. $S(t, x)$ is defined in terms of $T$, and let the only $1 / c$-periodic solution of

$$
W_{t}-W_{x x}+c W_{x}=\frac{3}{2}\left(\mathcal{F}_{1}[T]\right)^{3 / 2} \varphi(T), \quad W(t,-\infty)=Y_{u},
$$

be defined as

$$
W(t, x)=\gamma(x) Y_{u}+(1-\gamma(x)) T_{b}\left(Y_{u}, S_{u}\right)+\mathcal{F}_{2}[T] .
$$


The equation that we are solving is then

$$
\begin{aligned}
\left(\partial_{t}-\partial_{x x}+c \partial_{x}\right) T & =\left(Y_{u}+(1-\gamma)\left\langle S_{u}^{3 / 2}\right\rangle+\left(\mathcal{F}_{2}[T]-T\right) f(T)\right. \\
T(t, x) & =\phi_{0}(x)+O\left(e^{r x}\right) \quad(x \rightarrow-\infty) \\
T(t,+\infty) & =T_{b}\left(Y_{u}, S_{u}\right)
\end{aligned}
$$

As soon as we find a $1 / c$-periodic solution to 5.29 , we will be done.

Once the estimates are at hand, the rest of the proof of the theorem only amounts to putting 5.29] in the right functional form, so we are not going to dwell on it too much, all the more as it was detailed in Section 3. The unknown $T(t, x)$ is best looked for in the form

$T(t, x)=\left(1-\gamma(x) T_{b}\left(Y_{u}, S_{u}\right)+\left(\gamma(x)\left(\phi_{0}(x)+e^{r x}\right)+(1-\gamma(x)) e^{-\rho x}\right) u(t, x)\right.$

where the exponent $r$ is defined in 5.26 and the exponent $\rho$ is, say, less than $\rho_{0} / 2$ where $\rho_{0}$ is defined in Lemma 5.3 This complicated-looking expression simply says that we wish $T$ to be asymptotic to $\phi_{0}(x)$ as $x \rightarrow-\infty$, and to converge to $T_{b}\left(Y_{u}, S_{u}\right)$ at some small exponential rate. The equation for $u(t, x)$ therefore reads

$$
u_{t}+L u=f_{0}\left(x, u, u_{x}\right)+\phi_{1}(x)+\frac{\left(\mathcal{F}_{2}[T]-T\right) f(T)}{\gamma(x)\left(\phi_{0}(x)+e^{r x}\right)+(1-\gamma(x)) e^{-\rho x}} .
$$

where the quantities $L, f_{0}$, and $\phi_{1}$ have the following features.

1. The operator $L$ is defined as

$$
\begin{aligned}
L & =-\frac{d^{2}}{d x^{2}}+a(x) \frac{d}{d x}+b(x), \\
a(x) & =\gamma(x)(c-2 r)-(1-\gamma(x))(c-2 \rho), \\
b(x) & =\gamma(x)\left(Y_{u}+c r-r^{2}\right)+(1-\gamma(x))\left(T_{b}\left(Y_{u}, S_{u}\right)-c \rho-\rho^{2}\right) .
\end{aligned}
$$

Notice that $b(x)$ is controlled from below by a positive constant. By the maximum principle we have therefore the inequality

$$
\left\|e^{-L / c}\right\|_{\mathcal{L}(U C(\mathbb{R}))}<1 .
$$

2. The function $f_{0}$ is smooth in all its variables; moreover we have

$$
\forall x \notin[0,1], \forall(u, p) \in \mathbb{R}^{2}, \quad f(x, u, p)=0 .
$$

3. There is $\delta_{0}$ depending only on the data such that

$$
\forall x \in \mathbb{R}, \quad\left|\phi_{1}(x)\right| \leq C e^{-\delta_{0}|x|} .
$$

4. Recall that $T$ is (for short) defined by equation 5.30). From Proposition 5.2. Lemma 5.3 and Proposition 5.4 there exists $\delta_{1}<\min \left(r, \rho_{0} / 2\right)$ such that if $T-\left(Y_{u}-\left\langle S_{u}^{3 / 2}\right\rangle\right)(1-\gamma)$ 
belongs to $X_{r, \delta_{0}}$, then, for some $C$ depending on the $X_{r}$ norm of $T-\left(Y_{u}-\left\langle S_{u}^{3 / 2}\right\rangle\right)(1-\gamma)$,

$$
\forall x \in \mathbb{R}, \quad\left|\frac{\left(\mathcal{F}_{2}[T]-T\right) f(T)}{\gamma(x)\left(\phi_{0}(x)+e^{r x}\right)+(1-\gamma(x)) e^{-\rho x}}\right| \leq C e^{-\delta_{0}|x|} .
$$

Consequence: let $\mathcal{K}[u]$ denote the right side of (5.31). Then the operator $\mathcal{F}$ acting on $C_{\text {per }}^{\alpha, \alpha / 2}$ and defined by $\mathcal{F}[u]=e^{-L / c} \mathcal{K}[u]$ is compact in $C_{\text {per }}^{\alpha, \alpha / 2}$.

Now, it suffices to perform the homotopy consisting in replacing once again $S_{u}$ by $\tau S_{u}$, while keeping $Y_{u}$ fixed. For $\tau=0$, this reduces to the study of the operator $-d^{2} / d x^{2}$ $+c d / d x-f^{\prime}\left(\phi_{0}\right)$, which is an isomorphism between its domain and the set of all functions $u$ decaying like $e^{r x}$ on the left side; see [13]. This implies the existence result.

\section{Extensions}

Clearly, in this paper we have not discussed the thermo-diffusive propagation of spray flames in its full generality, although we believe that we have captured some of its main features in the study that we have presented. Some extensions of the theory developed so far can be thought about; some of them are simple generalizations; others seem less obvious to us. Let us list three of them.

- Holes in the distributions of droplets at $-\infty$. We have only treated droplet distributions at $-\infty$ that never vanished. This is truly a convenience assumption, which is only needed in the lower bound for the velocity in the ignition temperature case. A more accurate proof would have revealed that what matters is $\left\langle S_{u}\right\rangle$. This generalization is omitted.

- Polydisperse sprays. A more general description of the spray would describe the droplet distribution by a probability density $f(t, x, s)$ accounting, roughly speaking, for the number of droplets that, between the times $t$ and $t+d t$, and between the positions $x$ and $x+d x$, have sizes comprised between $s$ and $s+d s$. The governing equations are then

$$
\begin{aligned}
& T_{t}-T_{x x}=Y f(T) \\
& Y_{t}-Y_{x x}=-Y f(T)+\varphi(T) \int_{0}^{+\infty} s^{3 / 2} f(t, x, s) d s \\
& f_{t}-\partial_{s}(\varphi(T) H(s) f)=0 .
\end{aligned}
$$

Here $H(s)$ is the Heaviside function. Such a general spray is said to be polydisperse, as opposed to the monodisperse case that we have treated so far. The system we treat has two simplifications relative to (6.1): first, we have replaced $H(s)$ by the function identically equal to $s$ to avoid dealing with interfaces. We have also assumed that the distribution $f(t, x, s)$ has the form $\delta_{s=S(t, x)}$ as we consider the monodisperse case.

Although 6.1 looks much more formidable than (1.1), the investigation of travelling waves is essentially the same as for $[1.3$ (see [7]) - the relevant quantity to be considered is the maximal size of the droplets at $-\infty$. We expect that this is also the case for the pulsating fronts. 
- Several space dimensions. What prevents us from going to several space dimensions is the lap number decay-which is, in Section 4, replaced by another typically 1D result. Most certainly, one could by-pass the use of this result.

A less obvious point to study is what happens when there is no gaseous fuel at $-\infty$, i.e. $Y_{u}=0$. The main question to be answered is: is there an unbounded range of possible velocities? Preliminary computations of the travelling wave problem seem to indicate that this is impossible, and that the system would be qualitatively close to the one with ignition temperature. This, however, needs a more serious study than these considerations. Also, a complete study of the burning rate, in the spirit of [8] or [12], would be very welcome. This might not be an easy task, for we would have to drop the pulsating wave assumption - an assumption that we have heavily used in several crucial instances. In particular, we still do not know how to derive an upper bound for the enthalpy, although sharp upper bound results for the purely gaseous thermo-diffusive system, such as [10], might help. We hope to give (at least partial) answers to these last two questions in the future.

Acknowledgements. The second and third authors gratefully acknowledge several invitations by the Department of Mathematics of the University of Chicago, where the main ideas of this paper were discussed. This research was also supported in part by the ASCI Flash center at the University of Chicago under DOE contract B341495. PC was partially supported by the NSF grant DMS0202531, LR by NSF grant DMS-0203537, ONR grant N00014-02-1-0089 and an Alfred P. Sloan Fellowship.

\section{References}

[1] Angenent, S.: The zero set of a solution of a parabolic equation. J. Reine Angew. Math. 390, 79-96 (1988) Zbl 0644.35050 MR 0953678

[2] Aronson, D. G., Weinberger, H. F.: Nonlinear diffusion in population genetics, combustion, and nerve pulse propagation. In: Partial Differential Equations and Related Topics, Lecture Notes in Math. 446, Springer, Berlin, 5-49 (1975) Zbl 0325.35050 MR 0427837

[3] Aronson, D. G., Weinberger, H. F.: Multidimensional nonlinear diffusion arising in population genetics. Adv. Math. 30, 33-76 (1978) Zbl 0407.92014 MR 0511740

[4] Berestycki, H., Larrouturou, B.: Quelques aspects mathématiques de la propagation des flammes prémélangées. In: Collège de France Seminar, Vol. X (Paris, 1987-1988), Pitman Res. Notes Math. Ser. 220, Longman Sci. Tech., Harlow, 65-129 (1991) Zbl 0755.35090 MR 1131819

[5] Berestycki, H., Nicolaenko, B., Scheurer, B.: Travelling wave solutions to combustion models and their singular limits. SIAM J. Math. Anal. 16, 1207-1242 (1985) Zbl 0596.76096 MR 0807905

[6] Berestycki, H., Hamel, F.: Front propagation in periodic excitable media. Comm. Pure Appl. Math. 55, 949-1032 (2002) Zbl 1024.37054 MR 1900178

[7] Berthonnaud, P., Domelevo, K.: Travelling wave solutions for the equations of onedimensional spray flames. Preprint (2002)

[8] Constantin, P., Kiselev, A., Oberman, A., Ryzhik, L.: Bulk burning rate in passive-reactive diffusion. Arch. Ration. Mech. Anal. 154, 53-91 (2000) Zbl 0979.76093 MR 1778121 
[9] Henry, D.: Geometric Theory of Semilinear Parabolic Equations. Lectures Notes in Math. 840, Springer, New York (1981) Zbl 0456.35001 MR 0610244

[10] Herrero, M. A., Lacey, A. A., Velázquez, J. J. L.: Global existence for reaction-diffusion systems modelling ignition. Arch. Ration. Mech. Anal. 142, 219-251 (1998) Zbl 0909.35063 MR 1636529

[11] Kavian, O.: Introduction à la théorie des points critiques et applications aux problèmes elliptiques. Mathématiques \& Applications (Berlin) 13, Springer, Paris (1993) Zbl 0797.58005 MR 1276944

[12] Kiselev, A., Ryzhik, L.: An upper bound for the bulk burning rate for systems. Nonlinearity 14, 1297-1310 (2001) Zbl 0988.35080 MR 1862822

[13] Sattinger, D. H.: On the stability of waves of nonlinear parabolic systems. Adv. Math. 22, 312-355 (1976) Zbl 0344.35051 MR 0435602

[14] Uchiyama, K.: The behavior of solutions of some nonlinear diffusion equations for large time. J. Math. Kyoto Univ. 18, 453-508 (1978) Zbl 0408.35053 MR 0509494

[15] Vakulenko, S., Volpert, V.: New effects in propagation of waves for reaction-diffusion systems. Asymptotic Anal. 38, 11-33 (2004) Zbl 1073.35119 MR 2060618

[16] Williams, F. A.: Combustion Theory. Benjamin-Cummings, Menlo Park, NY (1985)

[17] Xin, J.: Existence of planar flame fronts in convective-diffusive periodic media. Arch. Ration. Mech. Anal. 121, 205-233 (1992) Zbl 0764.76074 MR 1188981 